\title{
METODOLOGÍAS CUALITATIVAS DE LO SENSIBLE EN CONTEXTOS DE SALUD PÚBLICA
}

\author{
QUALITATIVE METHODOLOGY OF SENSITIVE TOPICS IN PUBLIC HEALTH CONTEXTS
}

\author{
METODOLOGIAS QUALITATIVAS DO SENSITIVO NO CONTEXTO DE SAÚDE PÚBLICA
}

\author{
Mirliana Ramírez-Pereira \\ Departamento de Enfermería, Universidad de Chile. Santiago, Chile. \\ Departamento de Salud Pública, Facultad de Medicina, Universidad Católica del Norte. Antofagasta, Chile. \\ mirliana@uchile.cl \\ ORCID: 0000-0003-1439-4162 \\ DOI: https://doi.org/10.22235/ech.v6iEspecial.1447 \\ Recibido: $15 / 06 / 2017$ \\ Aceptado: 22/08/2017
}

\section{RESUMEN}

La subjetividad siempre se encuentra presente en las metodologías cualitativas, en que se vinculan las emociones, sentimientos, cultura e historia de los participantes e investigadores. Esto es especialmente relevante en el área de la salud, donde lo sensible está presente en muchos de los temas estudiados. Según Lee los temas sensibles son aquellos en que la investigación invade la esfera privada, escarba dentro de alguna experiencia personal profunda, hace referencia a la desviación o control social, afecta a los intereses de personas poderosas o el ejercicio de la coerción y la dominación. Este artículo se divide en dos apartados; el primero tiene relación con la fundamentación teórica del estudio de temas sensibles en salud pública, desde la perspectiva de los participantes y del equipo de investigación, relevando la importancia de la seguridad e integridad física, psíquica, moral y legal de todos los actores involucrados. En la segunda parte, la autora reflexiona en torno a su experiencia en la investigación en temas sensibles, presentando algunas estrategias de abordaje del trabajo de campo. El objetivo de esta investigación fue describir la producción del conocimiento en relación a la investigación de temas sensibles en salud.

Palabras clave: Investigación Cualitativa, Salud Pública, Ética, Relaciones Investigador-sujeto.

\section{ABSTRACT}

Subjectivity is always present in qualitative methodologies where emotions, feelings, culture and background of participants and researchers are involved. This is especially relevant in the health area, where sensitivity is present in many of the topics addressed. According to Lee, sensitive topics are those where research invades the private sphere, digs into some deep and personal experiences, addresses deviation or social control, and affects the interests of powerful people and/or the execution of coercion and domination. The following article is divided in two parts: the first one is related to theoretical foun- dations of the study of sensitive topics in public health, from the perspective of participants and the research team, revealing the importance of safety and physical, psychological, moral and legal security of all the members involved. In the second part, the author reflects upon her experience in investigation of sensitive topics, and, finally, methodological strategies are proposed to address the study of these subjects.

Keywords: Qualitative Research, Public Health, Ethics, Researcher-subject Relationship.

\section{RESUMO}

A subjetividade está sempre presente nas metodologias qualitativas, onde se vinculam as emoções, os sentimentos, a cultura e a história dos participantes e pesquisadores. Isto é especialmente relevante na área da saúde, onde o sensível está presente em muitos dos temas abordados. Conforme com Lee, se considera uma questão sensível quando a pesquisa invade a esfera privada ou examina, indaga alguma experiência pessoal profunda. Refere-se ao desvio ou controle social, afeta os interesses de pessoas poderosas ou o exercício da coerção e dominação, se lida com coisas sagradas para as pessoas estudadas e não querem ser profanadas. Ele é dividido em duas seções; a primeira está relacionada com a fundamentação teórica do estudo de questões sensíveis na saúde pública, a partir da perspectiva dos participantes e da equipe de investigação, relevando a importância da segurança e da integridade física, mental, moral e legal de todos os atores envolvidos. Na segunda parte, o autor reflete sobre sua experiência em pesquisa sobre questões sensíveis, apresentando algumas estratégias de enfrentamento trabalho de campo.

Palavras-chave: Pesquisa Qualitativa, Saúde Pública, Ética, Relações Pesquisador-sujeito. 


\section{INTRODUCCIÓN}

Este artículo nace de la iniciativa de la Escuela de Verano en Metodologías Cualitativas para la Transformación Social en Zonas Fronterizas de la Universidad de Tarapacá, Chile, como una forma de diseminar y poner a disposición de la mayor cantidad de interesados las clases y talleres desarrolladas en esa oportunidad en la ciudad de Arica. En mi calidad de enfermera y académica, el tema que presenté fue "Metodologías cualitativas de lo sensible en contexto de salud pública en Chile". ${ }^{1}$

Para la elaboración de la clase-taller en primer lugar realicé una introspección respecto a mis vivencias como enfermera y mujer latina que investiga temas sensibles; me pregunté qué emociones surgieron de esa experiencia, de qué manera abordé las diferentes temáticas que en muchas ocasiones eran conocidas por mí sólo como aproximaciones teóricas y más aún, como estadísticas, sin asociar ni conocer las historias de las personas que acompañan a las cifras. Luego de este proceso realicé una búsqueda bibliográfica, revisando textos clásicos y publicaciones recientes, y por último diseñé la clase y el taller con la intención de que recogiera esta amalgama entre experiencia vivida y conocimiento científico. Para la escritura de este documento retiré la palabra "Chile", de forma de poder incluir experiencias compartidas como seres humanos. La pregunta de investigación fue: ¿Qué conocimientos existen con respecto a la investigación de temas sensibles en salud? Y el objetivo fue describir la producción de conocimientos en relación a la investigación de estos temas.

\section{METOdOLOGÍA}

La metodología utilizada en esta investigación fue autoetnográfica. Una de las nuevas vías para contribuir al cuidado humanista y centrado en la persona y su familia es la autoetnografía interpretativa, la que se comprende como el uso de la experiencia y escritura personal para comentar las vivencias y prácticas culturales, denunciar la vulnerabilidad y crear relaciones recíprocas con la audiencia en atención a lograr una respuesta (1).

Constó de dos etapas. En la primera parte se explica la metodología de la revisión bibliográfica integrativa y en la segunda la autora realiza una reflexión respecto a su propia experiencia de investigadora; para esto realizó un proceso de introspección plasmado en la escritura para descubrir su epifanía y camino para llegar a investigar estos temas.

La pregunta de investigación planteada fue ¿Qué conocimientos existen con respecto a la investigación de temas sensibles en salud? El objetivo fue describir la producción de conocimiento en relación a la investigación de temas sensibles en salud. Con este fin se revisó literatura disponible en texto completo en las bases de datos Scielo, Scopus, Web of Science y

1 Escuela de Actualización en Metodología Cualitativa, organizada por el Escuela de Psicología y Filosofía de la Universidad de Tarapacá en la ciudad de Arica los días 12 y 13 de enero del año 2017.
EBSCOhost research database, además de literatura disponible en libros de investigación en el área.

\section{RESULTADOS}

El estudio de temas sensibles emerge como consecuencia de la comprensión de la salud desde la integralidad del ser humano, incluyendo aspectos psicosociales, físicos y espirituales. Esto se ha acompañado con un incremento de la investigación en temas tales como vivir con SIDA, padecimiento de enfermedades altamente contagiosas, adicciones, género, sexo, marginalidad, inmigración, violencia, entre otros, los que según la literatura disponible son considerados "temas sensibles" (2).

Según Lee los temas sensibles son aquellos tópicos en que la investigación invade la esfera privada examinando alguna experiencia personal profunda, hace referencia a la desviación o control social, afecta a los intereses de personas poderosas o el ejercicio de la coerción y la dominación, y por último, aquellos temas que lidian con cosas sagradas para los participantes y que no quieren que sean profanadas (2).

Por otro lado, Sieber y Stanley definen el estudio de temas sensibles como aquellos en los cuales podría haber consecuencias o implicaciones potenciales en los participantes en la investigación (3).

La investigación en los mundos y experiencias privadas de las personas, en que se evocan respuestas emocionales complejas y se revelan problemas íntimos, obliga a los científicos a profundizar en áreas en que se desconocen las implicaciones emocionales, físicas y espirituales para los participantes a mediano y largo plazo, las cuales afectan a usuarios, investigadores, transcriptores y ayudantes de investigación (4).

En el trabajo con temas sensibles se debe considerar la identificación de aspectos que se desean mantener en secreto; también el hecho de que hay personas, instituciones o en ocasiones el mismo Estado a los que les interesa mantener oculta cierta temática. Se recomienda que el investigador conozca a cabalidad el tema en estudio y diseñe estrategias para acercarse a los participantes, proteger la integridad física, emocional, social y legal de todos los involucrados y además de la protección de la información recabada (2).

Los antecedentes históricos del estudio de temas sensibles comienzan con las investigaciones de los sociólogos de la Escuela de Chicago, quienes, sin tener clara conciencia del impacto de sus investigaciones, tuvieron acceso a las vidas privadas de las personas que tomaban parte de sus estudios (2).

Estas investigaciones fueron continuadas con los movimientos de liberación femenina de la década de los sesenta, quienes, junto con realizar campañas contra la visión de la mujer como un objeto y la violencia ejercida contra ellas, desarrollaron métodos de investigación de tipo no-hierático entre investigador e investigado. Estos métodos, utilizados en la actualidad, estudian las relaciones de poder, dominación, violaciones, desórdenes alimenticios, entre otros (4-6). Las investigaciones 
feministas desarrollan relaciones interpersonales particularmente humanas por la naturaleza íntima de las entrevistas y conversaciones (6).

El estudio de temas sensibles habitualmente se realiza desde las metodologías cualitativas y en ese sentido, para trabajar con estas temáticas, los investigadores han recorrido un camino que los ha conducido a éstas. A modo de ejemplo, Berger refiere que se acercó a las metodologías cualitativas y a los temas sensibles cuando estudió a su propia familia, sobreviviente del holocausto. Esta área de investigación se consolidó con su trabajo en discapacidades luego del diagnóstico de su hija con parálisis cerebral (7).

La entrevista es una de las técnicas más usadas en la investigación de temas sensibles. DeMarrais y Tisdale, citados por Ashton, identificaron la importancia de crear un espacio en que la persona pueda expresar sus emociones (8). Según ellos, si se logra crear este espacio es posible compartir experiencias íntimas en entrevistas en profundidad. Elmir recomienda que previo a la entrevista se haya contactado telefónicamente al participante, para así conseguir cierto grado de rapport. Por otro lado, se sugiere que la comunicación sea bidireccional y que el investigador también comparta algo de su historia, además de no presionar con el tiempo al participante (9). Petersen refiere que el solo hecho de narrar una historia es emancipatorio para el participante, porque le permite expresar sus sentimientos, ideas y emociones y dar pie a un proceso de liberación (10).

Desde el punto de vista ético, un aspecto fundamental es la mantención del anonimato de los participantes. Janesick menciona que es recomendable preguntar al participante cómo quiere ser llamado en la investigación, sea con seudónimos, iniciales o nombres simbólicos, los que pueden ayudar a comprender sus narrativas y cumplir así el criterio de anonimato (11).

Con respecto a las dificultades en el estudio de temas sensibles, Lee menciona que el investigador se enfrenta a problemas metodológicos y técnicos, entre los cuales se encuentran la conceptualización del objeto de estudio, definición y acceso a los participantes, desconfianza, encubrimiento de la información e inseguridad de todos los involucrados (2). También puede presentar sintomatología física, especialmente en el trabajo de campo, en la que incluyen cefalea, insomnio, pesadillas y molestias gastrointestinales, siendo más frecuente en los investigadores que recién comienzan. Con el fin de minimizar estos efectos se recomienda discutir a priori las posibles consecuencias materiales y psicosociales que tendría la investigación (11).

A pesar de los inconvenientes en la realización de estas investigaciones, Dickson-Swift considera que los científicos deben estudiar estas temáticas por la responsabilidad ética y social de la ciencia (12).

Continuando con la idea del párrafo anterior, Gilbert en Dickson-Swift plantea que la combinación de temas con una alta carga sensible y con contacto prolongado en el tiempo puede derivar en una pérdida de los límites en la relación de los par- ticipantes e investigadores, por lo cual estos límites deben ser constantemente negociados y renegociados, intentando lograr un equilibrio entre los costos y beneficios de estar más o menos cerca de los investigados (12).

Es interesante mencionar una investigación realizada en Australia en la cual se entrevistaron a $\mathbf{3 0}$ investigadores que estudian temas sensibles, como, por ejemplo, personas en situación de calle, sexualidad, cáncer, abuso de drogas y alcohol, violencia, muerte y suicidio. Utilizando la teoría fundada, los investigadores identificaron las categorías siguientes: ser un investigador "profesional", ¿investigación o terapia?, límites entre amistad e investigación, e implicaciones potenciales de un inadecuado manejo de estos límites. En la categoría ser un investigador profesional, los participantes refirieron que se esfuerzan en ser profesionales, manteniendo límites seguros en su relación con los investigados, con un distanciamiento emocional de la persona. Sin embargo, también refirieron que la investigación cualitativa lleva inherente la conexión emocional entre personas. Con respecto a la categoría ¿investigación o terapia? refieren que en ocasiones les resulta difícil diferenciar en las entrevistas cuándo el objetivo es investigar, versus cuándo es realizar terapia o consejería, sintiendo que inevitablemente su intervención tiene un efecto terapéutico (13).

En relación a los límites entre amistad e investigación, los investigadores participantes refirieron que a menudo se produce una cercanía importante con las personas investigadas, acercándose a la amistad; esto se complejiza cuando se concluye la investigación y es momento de despedirse y cortar todo nexo con los participantes. Las implicaciones potenciales de un inadecuado manejo de límites se traducen en agotamiento emocional, con el consiguiente riesgo de síndrome de burnout (14).

Manders observó la trayectoria de los investigadores en una comunidad Sikh Punjabi en Inglaterra. El objetivo de la investigación fue explorar la factibilidad de desarrollar un programa comunitario para el tratamiento del alcoholismo dentro de la comunidad, en la cual se considera normal el consumo de alcohol en exceso por parte de los hombres. Como conclusiones de la experiencia investigativa, los autores refieren la importancia de manejar el idioma del grupo en estudio, obtener un rapport adecuado, llegar a convertirse desde un extraño a un "profesional que ayuda"; también recomiendan dejar de lado el uso de etiquetas asociadas a sus costumbres en los participantes. De igual manera sugieren una actitud de compromiso, honestidad y paciencia en lo que respecta a la suspensión de parte de los participantes de citas sin previo aviso (15).

Los autores antes mencionados coinciden en la importancia de un buen rapport, empatía, honestidad, dejar fuera los prejuicios, tener conocimiento acabado del objeto de estudio; incluyen también conocer costumbres y hábitos, el idioma en el caso de que la investigación se realice en una comunidad con una lengua distinta a la propia, resguardar aspectos éticos, legales y de seguridad inherentes al estudio de temas sensibles. También recomiendan conversar con todo el equipo investigador respecto de los significados que tiene para cada uno el estudio del tema en cuestión. 
A continuación, presento mi experiencia como investigadora en temas sensibles, con algunas recomendaciones respecto al trabajo de campo en estos temas.

\section{DISCUSIÓN}

Busco el mejor momento para escribir, aquel momento en que las ideas y recuerdos fluyen; cierro los ojos y recuerdo, intentando conectarme también con las emociones que surgen del estudio de temas sensibles.

Mi vinculación con la investigación de temas sensibles comenzó con el ingreso a estudiar Enfermería, en que la aproximación del cuidado siempre se hace desde la relación persona a persona. Cuando confeccionaba la historia clínica de los pacientes me interesaban sus vivencias y experiencia, me surgían interrogantes respecto a los factores personales y socioculturales que los afectaba. Sin embargo, revisando mi historia de vida, mi epifanía ocurrió en el momento cuando siendo aún estudiante pude asistir a un curso de Medicina Social.

Según Denzin, la epifanía es un momento de la experiencia vivida que uno puede identificar como un punto de inflexión en la comprensión de uno mismo y de la propia relación con el mundo. Así, la epifanía obliga al investigador a regresar y explorar ese momento que altera la vida. La urgencia emocional inducida por la epifanía convierte así la instrucción metodológica -que hay que volver constantemente a ese momento- en un imperativo, lo que significa que hay que reexaminar constantemente la epifanía (1).

Los temas que tenían relación con pobreza, desigualdad, dictadura, miedo, no se discutían abiertamente en las escuelas de Enfermería. La herencia de la dictadura militar limitaba la enseñanza a una visión fuertemente biomédica de la salud. En ese curso comprendí que las enfermedades eran mucho más que agentes biológicos o estilos de vida, sino que estaban impregnadas de hambre, miseria e inequidad. Comprendí que la tuberculosis no era sólo el bacilo de Koch, sino que estaba entretejida con pobreza, hambre, hacinamiento, tristeza y miedo. En el momento de comprender estas relaciones pude conectar muchos hechos de mi vida que se resumían en una infancia en dictadura, con mi madre al borde de la muerte por tuberculosis, hospitalizada y recibiendo antibióticos por un año.

Luego de mi ejercicio profesional como enfermera especialista en nefrología, enfocada en personas con enfermedad renal crónica, los estudios de postgrado partiendo con un Master en Psicología Comunitaria y luego con el Doctorado en Enfermería, fueron introduciéndome en el estudio de temas sensibles. Mi primer acercamiento ocurrió cuando estudié las representaciones de la enfermedad renal crónica en personas en peritoneo diálisis; fueron horas de entrevistas en las que, según Valles, el entrevistado debe percibirla como una conversación, sin que se dé cuenta de la estructura de la interrogación, el orden de las preguntas o los objetivos del entrevistador (16). En esa investigación pude observar y sentir todo el sufrimiento asociado a la enfermedad renal, cuando las personas en diálisis me relataban su historia en que se mezclaban emociones como rabia, culpa, resignación, miedo y frustración. Por mi parte, podía sentir incluso físicamente el sufrimiento; a veces sentía deseos de llorar junto al paciente, pero le tomaba la mano y le pedía que descansara y que si necesitaba detener la entrevista lo podía hacer. Pero contrariamente a lo que pensaba que podía ocurrir, continuaba con la entrevista y se despedía agradeciendo la oportunidad de hablar y ser escuchado.

Con mi desarrollo académico centrado en la investigación cualitativa en salud continué investigando temas sensibles. Una de las experiencias que me parece interesante destacar fue cuando guié una tesis del Master en Salud Pública, en que se estudiaba la experiencia de hombres que tienen sexo con hombres en cuartos oscuros, es decir, espacios donde hay contacto sexual grupal entre desconocidos, sin precauciones ni protección de ningún tipo. Aunque yo no realicé las entrevistas participé en el análisis, por lo que debí leer y releer los textos en muchas ocasiones. Los entrevistados se extendieron en detalles y prácticas sexuales no tradicionales, las cuales involucraban adolescentes que, aunque mayores de edad, se prostituían por necesidades económicas. Yo me considero una persona libre de prejuicios; sin embargo, esas lecturas me afectaron físicamente, habiendo momentos en que, como describe Cowles, sufrí de cefalea y náuseas, por lo cual debía detenerme, salir al jardín y respirar para dejar de lado los sentimientos de impotencia y rabia que me producía y aún me produce la explotación sexual (12).

En otra oportunidad pude vivenciar lo que significaba estudiar temas sensibles en el caso de las instituciones. Quise investigar, junto a estudiantes de último año, las causas de deserción de alumnos de enfermería en una universidad en Chile, la que alcanzaba a un $30 \%$ en los dos primeros años. Cuando analizamos los resultados, emergió información que mostraba que los estudiantes desertaban por sentirse maltratados y humillados por las profesoras que los supervisaban en experiencia clínica, que eran enfermeras clínicas contratadas para estos efectos; también renunciaban por aspectos relacionados con las metodologías de enseñanza; consideraban que el nivel de exigencia en las prácticas clínicas no tenía relación con los conocimientos teóricos que se les entregaban, siendo estos últimos de inferior calidad. Como consecuencia de estos resultados, alejados del espíritu de la Escuela de Enfermería, se citó a reunión urgente del consejo; se decidió que en adelante todas las investigaciones debían pasar, además del comité de Ética Clínica, por la evaluación de las profesoras de la carrera y por último se suspendió por ese año la presentación pública de las tesis de grado. El equipo de investigadores no previó el impacto de los resultados; pensamos que sería un aporte para disminuir la deserción, estableciendo las mejoras correspondientes, lo que no ocurrió. Lee refiere que estos son temas sensibles ya que investigan el control social, en este caso, dado por la institucionalidad, afectando los poderes fácticos con el uso de la coerción y la dominación (2).

La investigación de temas sensibles me ha dejado varias enseñanzas, coincidiendo con los autores mencionados en este texto: 
- $\quad$ Es fundamental conocer el tema que se desea estudiar; para eso es necesario recurrir a la revisión de aspectos contextuales como son las estadísticas, marco legal y jurídico, normativas propias de los países; también es necesario revisar el estado del arte y las teorías que apoyen o sustenten el tema.

- $\quad$ Es necesario realizar un proceso individual respecto a las ideas, estereotipos y prejuicios que rodean el tema escogido. En fenomenología se le denomina "epoche", lo que designa la suspensión de nuestra creencia implícita de un mundo independiente de la conciencia (17).

- Las reuniones de equipo para conversar, discutir, sentir el tema en estudio son en extremo importantes, ya que permiten la toma de conciencia respecto a qué ocurre con cada uno frente a un tópico que humaniza y acerca al otro, en el cual se comparten historias de vida, muchas veces cargadas de sufrimiento.

- $\quad$ También es importante la presencia de informantes claves, que conozcan el funcionamiento de la comunidad, y los aspectos "no dichos" y que puedan servir de puente con los investigadores.

- $\quad$ Otro aspecto importante es tener un real interés no sólo en el tema a investigar, sino principalmente en las personas a las cuales esperamos poder apoyar con el estudio. Del honesto interés con el otro surge un rapport adecuado. Creo que los participantes perciben si es que el investigador está interesado en ellos como persona, más allá de los datos que se puedan obtener.

- $\quad$ También es necesario haber realizado los vínculos previamente para referencia a apoyo psicosocial en el caso de que participantes o investigadores lo requieran.

- Como se menciona en el texto, los límites de la relación pueden ser borrosos. Creo que, en ese caso, es necesario dejar en claro para los participantes y también para nosotros los investigadores que la relación finaliza al término de la investigación, darlo a conocer con respeto y honestidad. Sin perjuicio de lo anterior, creo que es posible continuar con algún contacto de persona a persona, cuidando los códigos profesionales.
- En ocasiones, el estudiar temas sensibles puede resultar sanador para el investigador, ya que es posible que lea su propia historia en la historia del otro.

\section{CONCLUSIONES:}

El estudio de temas sensibles requiere un crisol de conocimientos, emociones conscientes, sentimientos, compromiso, honestidad y en algunos casos compasión por sí mismo y por el otro. Sin embargo, la investigación de temas sensibles es más que eso, porque aunque usa el método científico en forma rigurosa, debe enfrentarse a la imprecisión de ser humanos, emergiendo las dificultades que puede significar el estudio de temas no tradicionales, al margen de lo establecido y en algunas ocasiones fuera de la legalidad.

Cuando se estudian este tipo de temas, el investigador debe exponer en el camino metodológico las dificultades y limitaciones con las pudiera haberse encontrado; sin embargo, esto rara vez ocurre y los artículos publicados sólo muestran los resultados, sin que haya una presentación completa de lo que significa estudiar temas sensibles y de lo complejo que puede resultar esta amalgama de hechos y subjetividades.

Dada la complejidad de la realidad que se vive en el siglo XXI, en que el modelo neoliberal se encuentra arraigado en occidente y que persiste un descontento general en la ciudadanía, que exige sociedades más justas y tolerantes, es que se hace cada vez más urgente estudiar estos temas que desafían los cánones tradicionales de hacer ciencia y que nos conectan con la alteridad que está presente en todos los espacios.

\section{AGRADECIMIENTOS:}

Este artículo fue financiado por el Departamento de Enfermería de la Universidad de Chile con el proyecto "Espejuelos para el Género".

Cómo citar este artículo: Ramírez Pereira, M. Metodologías cualitativas de lo sensible en contextos de salud pública. Enfermeria (Montev.). [Internet]. 2017 Oct [citado xxxxx]; 6 25-30. Disponible en: https://doi.org/10.22235/ech. v6iEspecial.1447 


\section{REFERENCIAS}

1. Denzin N. Interpretive Autoethnography. California: SAGE; 2014.

2. Lee R. Doing Research on Sensitive Topics. California: Sage Publications; 1993.

3. Sieber JE, Stanley B. Ethical and professional dimensions of socially sensitive research. Am Psychol. 1988; 43 (1): 49-55.

4. McCosker H, Barnard A, Gerber R. Undertakin Sensitive Research: Issues and Strategies for Meeting the Safety Needs of All Participants. Forum Qual Soc Res. 2001. 2 (1): 1-14

5. Finch J. It s great to have someone to talk to: The ethics and politics of interviewing women. In: Bell C, and Roberts $\mathrm{H}$, Eds. Social researching: politics, problems, practice. London: Routledge \& Kegan Paul; 1984. p.79-88

6. Oakley A. Interviewing women: a contradiction in terms. In: Roberts H, Ed. Doing feminist research. London: Routledge \& Kegan Paul; 1981.p. 30-62

7. Berger R. Disability and Life History Research an Autoethnography of Qualitative Inquiry. Int Rev Qual Res. 2016. 9 (4): 472-488.

8. Ashton S. Researcher or nurse? Difficulties of undertaking semi-structured interviews on sensitive topics. Nurse Res. 2014; 22 (1): 27-31.
9. Elmir R, Schmied V, Jackson D, Wilkes L. Interviewing people about potentially sensitive topics. Nurse Res. 2011; 19(1): 12-16

10. Petersen A J. Research with individuals labeled "other": Reflections on the research process. Disabil Soc.2011; 26:293-305.

11. Janesick V. Contemplative Qualitative Research. Walnut Creek (California): SAGE; 2015.

12. Cowles K. Issues in Qualitative Research on sensitive topics. West J Nurs Res.1988; 10(2): 163-179.

13. Dickson - Swift V, Lyn E. Undertaking Sensitive Research in the Health and Social Sciences: Managing Boundaries, Emotions and Risks. New York: Cambridge University Press; 2008.

14. Dickson-Swift V, James E, Kippen S. Blurring Boundaries in Qualitative Health Research on Sensitive Topic. Qual Health Res. 2006; 16(6): 853-871.

15. Manders G, Galvani S. Learning from the Research Process: Discussing Sensitive Topics as a Cultural Outsider. Social Work Education. 2015; 34 (2): 199-212.

16. Valles M. Técnicas cualitativas de Investigación social. España: Síntesis; 1999.

17. Escudero JA. La actualidad de la fenomenología husserliana: superación de viejos tópicos y apertura de nuevos campos de exploración. Eidos. 2013; 18 (Jun-Jul): 12-45. 